\title{
Improving Performance of SDH/SONET-WDM Multilayer Networks using Weighted Integrated Routing
}

\author{
Marc Necker \\ Institute of Communication Networks and Computer Engineering \\ University of Stuttgart \\ Pfaffenwaldring 47, D-70569 Stuttgart \\ necker@ind.uni-stuttgart.de
}

\begin{abstract}
This work deals with routing schemes in SDH/SONET-WDM multilayer networks applying Synchronous Digital Hierarchy (SDH) or Synchronous Optical Network (SONET) on top of a dynamic photonic network in Wavelength Division Multiplex technology (WDM). The focus are integrated routing schemes which benefit from an integrated view of the SDH/SONET layer and the optical layer. Besides the comparison of known non-integrated and integrated methods, the new routing scheme Weighted Integrated Routing (WIR) is presented and its performance evaluated. In contrast to other known integrated routing schemes, WIR uses rating functions to evaluate different path alternatives and select the best route. In addition to the analysis of the different routing schemes, the dimensioning of network resources is treated.
\end{abstract}

\section{Introduction}

In optical networks, transmission rates have dramatically increased during the past years and dynamic provisioning of lightpaths will soon be available through an automated transport network control plane, e. g. ASTN [1]. Advanced node architectures which integrate electrical and optical layers will most likely be available in future systems. Assigning low-bandwidth electrical connections to high-speed optical lightpaths, also known as traffic grooming, is an important aspect in such networks, since small granularities are the major source of revenue for operators and provide service flexibility.

Routing on the electrical and optical layer is an essential component of every grooming strategy. Efficient transport of dynamic traffic demands of different granularities from the SONET/SDH hierarchy requires optimized multi layer routing and grooming algorithms [2]. Non-integrated routing schemes treat both layers separately while integrated schemes try to improve performance by combining both layers. Most approaches for integrated routing, e. g. [3] for SONET or [4] for IP, take advantage of complete knowledge about topology and occupancy of both layers, which is not supported by the overlay network model [5]. However, the overlay model is favored by operators as information on transport networks is very sensitive and should be kept secret. 
In this paper, Weighted Integrated Routing is presented which solves the dynamic grooming problem without the need for gathering full state information of both layers. Instead, the routing instance probes the SDH/SONET layer and the WDM layer for different path alternatives. In addition, a method for dimensioning the number of transponders in multilayer nodes is introduced.

The structure of this paper is as follows: In section 2, the network model and fundamentals on traffic grooming are introduced. In section 3 the Weighted Integrated Routing scheme is explained, followed by a discussion of transponder dimensioning in section 4. Finally, selected results of the performance evaluation are presented in chapter 5 .

\section{Multilayer Grooming and Routing Options}

The structure of a network node in the SDH/SONET-WDM network is shown in Fig. 1. On the optical layer, this model comprises an optical cross-connect (OXC), which is assumed to be non-blocking. Additionally, a certain number of wavelength converters allows for wavelength conversion without limitations. The electrical layer mainly consists of a non-blocking electronic cross-connect (EXC), which is able to switch electrical connections at arbitrary SDH/SONET granularities. The EXC thus allows for an effective grooming of electrical connections. The EXC and OXC are connected by $z$ transponders and receivers, respectively. On the optical layer, a node is connected to its neighboring nodes with a total of $w$ wavelengths in each direction.

In a centralized routing scheme, the routing control center (RCC) has to choose a path within the network for each connection request. It is therefore the

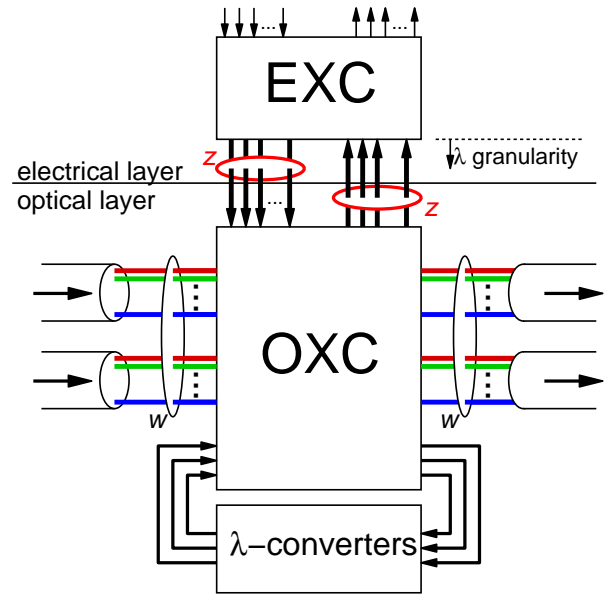

Figure 1. Multilayer node

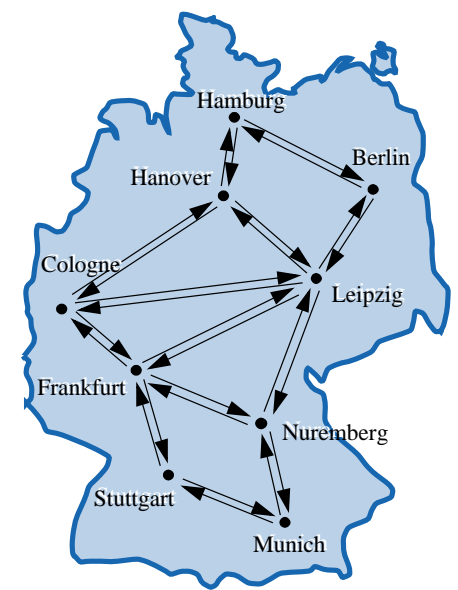

Figure 2. Network topology 
RCC's responsibility to efficiently groom the connections. Four basic grooming options can be identified:

\section{A Single-hop grooming on existing lightpath:}

The connection is assigned to one existing direct lightpath.

B Multi-hop grooming on existing lightpaths:

Routing takes place on the electrical layer by using more than one existing lightpath and switching the connection in the EXCs of intermediate nodes.

\section{Single-hop grooming on new lightpath:}

A new lightpath is set up between the source and the destination node. The connection request is routed on the optical layer via this new lightpath.

D Combined multi-hop grooming on new and existing lightpaths:

This is a combination of options $\mathrm{A}$ and $\mathrm{C}$. The connection request can be routed on both the electrical and optical layer by using a series of existing and new lightpaths.

Non-integrated routing schemes are capable of grooming on either existing or new lightpaths. In contrast to that, integrated routing schemes have enough information to also perform the combined grooming described in D. As a reference, two different non-integrated routing schemes will be considered, namely PreferOptical and PreferElectrical (TLRC in [3]). Both schemes belong to the class of overlay schemes [5] and differ in the order they apply the different grooming policies until one succeeds. In PreferOptical, the options are applied in the order A-C-B, whereas in PreferElectrical the order is A-B-C.

\section{$3 \quad$ Weighted Integrated Routing (WIR)}

\subsection{Basic Concept}

In contrast to the non-integrated routing schemes, WIR explores the different grooming strategies in parallel, including combined grooming. This implies that in any case all grooming strategies are considered, whereas non-integrated routing schemes apply the grooming strategies in a particular order and interrupt their search once a grooming strategy delivers a valid path. In WIR, each possible path that results from a grooming strategy is rated according to a set of criteria representing the path cost. Finally, the path with the best rating is chosen to route the connection. The process of rating paths is described in detail in sections 3.2 and 3.3 .

The ability of the routing scheme to perform combined grooming is an important design issue. On the one hand, the quality of the algorithm has a major influence on the performance of the routing scheme. On the other hand, it is much harder to find paths with combined grooming than applying grooming purely on the electrical or optical layer. Hence, it is a critical point in the design of the routing scheme to find an algorithm that delivers good combined grooming results at a reasonable complexity.

In [3], Zhu and Mukherjee use a reachability graph capturing all possible links for a given connection request between any two nodes for their proposed $S L R C$ 


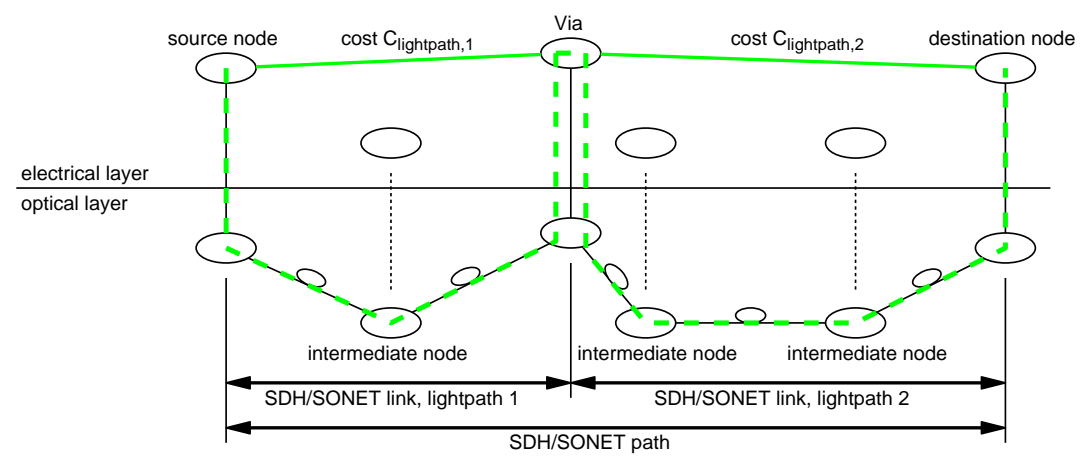

Figure 3. SDH/SONET path comprising two lightpaths

scheme. This approach has a constant high complexity. Here, an approach with variable complexity is presented. Combined grooming paths are searched along pre-calculated routes, for which the first $N_{\text {MaxAlt }}$ shortest completely link disjoint paths from source to destination are used. On each of these pre-calculated routes, up to $N_{\text {MaxVias }}$ intermediate nodes are allowed, in which the connection traverses the electrical layer. In the following, these nodes will also be referred to as Vias.

Fig. 3 illustrates how a connection might be switched within the network. In this example, the connection is routed along two lightpaths using one Via. A lightpath is represented as a logical link on the electrical layer and is also referred to as SONET/SDH link. The end-to-end path from source to destination node, on which the connection is routed, is termed SONET/SDH path. Eventually, any path alternative can be understood as an SDH/SONET path. Even a single lightpath is basically an SDH/SONET path comprising only one lightpath.

A route consisting of $N$ hops has $N-1$ vias. The $W I R$ algorithm starts by selecting one intermediate node and rating all four possibilities for combined grooming with respect to that node (new-new, new-existing, existingnew, existing-existing). In this intermediate node, the connection traverses the EXC whereas it is kept on the optical layer in all other intermediate nodes. That is, the node is a Via. Afterwards, the remaining intermediate nodes are selected one after another to be a Via, and rating information is gathered for any of the four possibilities for combined grooming.

If $N>2$, the algorithm additionally selects two intermediate nodes to be a via and rates all eight possibilities for combined grooming (new-new-new, new-new-existing, ... ) , eventually looking into all $\left(\begin{array}{c}N-1 \\ 2\end{array}\right)$ possibilities to select two intermediate nodes. Altogether, the search for combined grooming paths comprises all combinations of selecting up to $N_{\text {MaxVias }}$ intermediate nodes. The total number $M$ of tested path alternatives thus is:

$$
M=\left(\sum_{i=1}^{N_{\text {MaxAlt }}} \sum_{n=1}^{N_{\text {MaxVias }}}\left(\begin{array}{c}
N_{i}-1 \\
n
\end{array}\right)\right),
$$


with $N_{i}$ being the number of lightpaths of the $i$ th SDH/SONET path within the set of $N_{\text {MaxAlt }}$ shortest completely link disjoint paths. The parameter $N_{\text {MaxVias }}$ can dynamically be chosen for each connection request thus minimizing the algorithm's complexity (see below).

In contrast to other integrated routing schemes which require complete information of the electrical as well as the optical layer [3,4], WIR separately probes rating information on different paths from both layers and selects a path based on a cost $C$. This is a significant advantage for network operators, who often want to hide network details from customers. Note that the RCC only needs rating information from each individual layer. Non-existing or blocked lightpath requests can be signaled with an infinitely high cost.

Rating the different path alternatives is an essential part of this routing scheme and ultimately determines its performance. The following sections present methods to rate single lightpaths, and to calculate the rating of $\mathrm{SDH} / \mathrm{SONET}$ paths from the rating of the participating lightpaths.

\subsection{Rating an SDH/SONET link}

An SDH/SONET link occupies one transponder at the source node, one receiver at the destination node and one wavelength on each optical link of the lightpath. If necessary, wavelength converters are involved in intermediate nodes. Using the shortest path with $N_{\min }$ hops between each possible pair of nodes as a reference, the following two methods for calculating the $\operatorname{cost} C_{\text {lightpath }}$ of a lightpath were defined:

- Calculation using absolute path length

$$
C_{\text {lightpath }}=\left(N-N_{\text {min }}\right) \cdot W_{\text {OptHops }}+N_{\text {conv }} \cdot W_{\text {conv }} .
$$

- Calculation using relative path length

$$
C_{\text {lightpath }}=\left(\frac{N}{N_{\min }}-1\right) \cdot W_{\text {OptHops }}+N_{\text {conv }} \cdot W_{\text {conv }} .
$$

$N$ is the number of hops in the lightpath and $N_{\text {conv }}$ the number of occupied wavelength converters. $W_{\text {OptHops }}$ and $W_{\text {conv }}$ are weighting factors. The cost of the transponder at the source node and the receiver at the destination node are not considered, since these remain constant for every lightpath.

\subsection{Rating of an SDH/SONET path}

An SDH/SONET path comprises one or more lightpaths, which are connected on the electrical layer in the vias. The cost $C_{\mathrm{el}}$ of an $\mathrm{SDH} / \mathrm{SONET}$ path is made up of the cost of the necessary transponders and receivers in the vias and the

cost $C_{\text {lightpath,m }}$ of all participating lightpaths. Two methods which take into account the cost of these lightpaths were defined: 


\section{- Mean value calculation}

The weighted mean value of the rating of all participating lightpaths is included in the rating of the SDH/SONET path:

$$
C_{\mathrm{el}}=N_{Z} \cdot W_{\mathrm{PTE}}+\frac{W_{\text {lightpath }}}{N_{\text {Vias }}+1} \cdot \sum_{m=1}^{N_{\text {Vias }}+1} C_{\text {lightpath,m }} .
$$

\section{- Worst-Case calculation}

The weighted rating of the most expensive participating lightpath is included in the $\mathrm{SDH} / \mathrm{SONET}$ path rating:

$$
C_{\text {el }}=N_{Z} \cdot W_{\mathrm{PTE}}+W_{\text {lightpath }} \cdot \max _{m}\left(C_{\text {lightpath,m }}\right) .
$$

$N_{\text {Vias }}+1$ is the number of lightpaths an SDH/SONET path comprises. $W_{\text {PTE }}$ is the weighting factor for the cost of the Path Termination Equipment (PTE), i.e. lasers and diodes. Additionally, the weighting factor $W_{\text {lightpath }}$ allows for the weighting of all participating lightpaths.

In a practical implementation, the different grooming strategies will most likely be applied one after another instead of in parallel. If, for example, singlehop grooming on an existing lightpath delivers a path with a particular rating $C^{\prime}$, subsequent combined grooming makes sense only with $N_{\text {MaxVias }}=\left\lfloor\frac{C^{\prime}}{W_{\mathrm{PTE}}}\right\rfloor$. This property can be used to significantly lower the complexity of the algorithm by dynamically reducing the default value of $N_{\text {MaxVias }}$ for each connection request.

\section{Transponder and Receiver Dimensioning}

As mentioned before, the number of transponders or receivers $z_{k}$ at each node $k$ is a crucial design parameter of the system. If there are too few transponders, connections will already be blocked at the ingress or egress of the optical network. On the other hand, if there are too many transponders, resources (and thus money) will be wasted due to a low utilization of these units. Therefore, network planning has to precisely dimension the number of necessary transponders and receivers.

The traffic demand offered to the transponders at a multilayer node consists of three basic traffic types. 1 . The major portion is the node traffic $A_{\text {node }}$, i.e. the traffic generated by users at that particular node. 2 . The through traffic $A_{\text {transit }}$ describes the traffic switched by the EXC but not originating from or terminating at that node. 3 . The virtual traffic $A_{\text {virtual }}$ describes unused capacity on a wavelength. For example, if there are only STM-1, 7-STM-1 (VC-4-7v [6]) and STM-16 connections in an SDH-network, a wavelength can be occupied by two 7-STM-1 connections. In this case, the wavelength does not have enough free capacity to handle another 7-STM-1 or STM-16 connection, but only to handle two more STM-1 connections. This free capacity (which is "invisible" to some connection requests) can be modeled as virtual traffic $A_{\text {virtual }}$. The total offered traffic at the transponders of node $k$ thus is $A_{\text {transp }, k}=A_{\text {node }, k}+A_{\text {transit }, k}+A_{\text {virtual }, k}$. 
A simple but effective method to dimension the transponders is based on the assumption that $A_{\text {transit }, i}$ and $A_{\text {virtual }, i}$ are small compared to $A_{i}=A_{\text {node }, i}$. Assume further that most of the blocked connections are STM-16 connections (i.e. connections requiring an entire wavelength). The transponders can then be dimensioned using the well known Erlang-B-formula with one Erlang corresponding to a wavelength:

$$
B_{\text {transp }, \mathrm{k}}\left(A_{\text {transp }, k}, z_{k}\right)=\frac{\frac{A_{\text {transp }, k}^{z_{k}}}{z_{k} !}}{\sum_{i=0}^{z_{k}} \frac{A_{\text {transp }, k}^{i}}{i !}} .
$$

If the traffic $A_{\text {transp }, k}$ is known and the blocking probability $B_{\text {transp }, k}$ is given, one can calculate the number of transponders $z_{k}$ numerically.

For any given network topology, the number of transponders and receivers is the major variable cost factor. Therefore, the absolute cost was defined as the sum of all transponders in the network, which allows to effectively compare the performance of different routing schemes. For convenience, the relative cost was introduced as the absolute cost normalized by the sum of all $w_{k}$ at all nodes $k$, i.e. by the max. number of installable transponders. Since in our model the number of transponders equals the number of receivers at each node, only the transponders will be considered in the remainder of the paper.

Given a certain cost point, the transponders are distributed to the network nodes via the Erlang-B-formula, considering the offered traffic at each node. This is realized by an iterative algorithm, in which for each node $k$ the individual offered traffic $A_{\text {transp, } k}$ is used. $B_{\text {transp,k }}$ is chosen equal for all nodes. In each iteration step, $B_{\text {transp, } \mathrm{k}}$ is increased or decreased, until the desired cost point is reached. As a boundary condition, $z_{k}$ may not exceed $w_{k}$ at any node. That is, the actual number of transponders $z_{k}$ is calculated from the number of transponders as delivered by the Erlang Erlang-B-formula $z_{k, \text { Erlang }}$ as:

$$
z_{k}=\min \left(z_{k, \text { Erlang }}, w_{k}\right) .
$$

\section{Simulation Studies}

In order to study the performance of the different routing schemes, an event driven simulation tool was developed using the simulation library developed at the Institute of Communication Engineering and Computer Architecture of the University of Stuttgart [7].

All simulation studies were performed using a fictitious 9-node network of Germany, the topology of which is shown in Fig. 2. This network was introduced and dimensioned for static traffic demands in [8] using a traffic model which is based on the population and distance of the different nodes [9]. All links contain a certain number of fibers each holding 8 wavelengths. The ratio of offered traffic load in the dynamic scenario and traffic load used for static dimensioning defines the system load chosen to be $70 \%$ for all presented studies. On the optical layer, 
the dynamic routing scheme ADR3 [8] is applied, while the electrical layer uses a Dijkstra algorithm to compute the shortest route online.

The presented results were obtained without wavelength converters at each node. The influence of wavelength converters has been studied in depth in various publications (e.g. $[2,10])$ and a similar behavior can be expected in the presented environment. The weighting factors for WIR were set to 1 , and a worst-case path rating with a lightpath rating using relative path length was used.

A traffic mix consisting of $80 \%$ STM-1, $15 \%$ Gigabit-Ethernet (referred to as 7-STM-1 and transported as VC-4-7v in SDH [6], but without diverse routing) and 5\% STM-16 connection requests was used. The bandwidth of a wavelength was chosen to be STM-16. Connection requests arrive according to a Poisson process with exponential holding times.

Fig. 4 shows the blocking probability for different routing schemes. As a reference, the graph contains the performance of an opaque network with static point-to-point lightpath connections of adjacent nodes, which is only competitive at a relative cost of almost $100 \%$. The non-integrated routing scheme PreferOptical outperforms the scheme PreferElectrical by almost one order of magnitude. The integrated WIR achieves a request blocking probability, which is about one order of magnitude below the best non-integrated scheme and slightly below SLRC. There is a noticeable bend in all curves at a cost of about 0.55. For a cost below 0.55 , the request blocking probability is dominated by blocking at the transponders. For larger costs, there are sufficient transponders available and wavelength blocking dominates, i.e. it has no effect to further increase the number of transponders.

Fig. 5 shows the request blocking probability for different values of $N_{\text {MaxVias }}$. For $N_{\text {MaxVias }}=0$, there is no possibility for combined grooming, i.e. the WIR scheme is basically degenerated to a non-integrated scheme. Still, WIR outperforms the non-integrated schemes due to the use of rating functions. Increasing $N_{\text {MaxVias }}$ by one, which means including combined grooming at the lowest level,

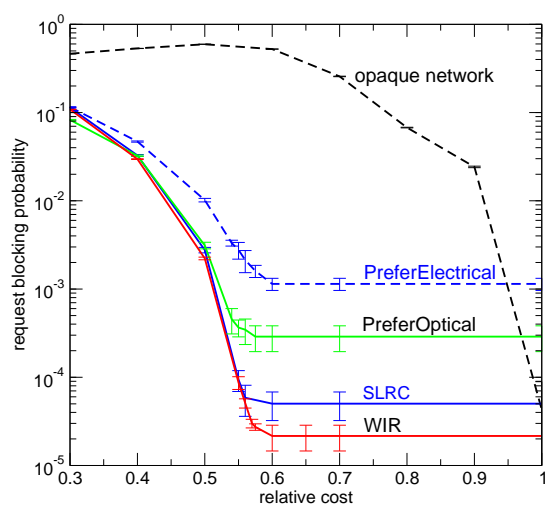

Figure 4. Request blocking probability

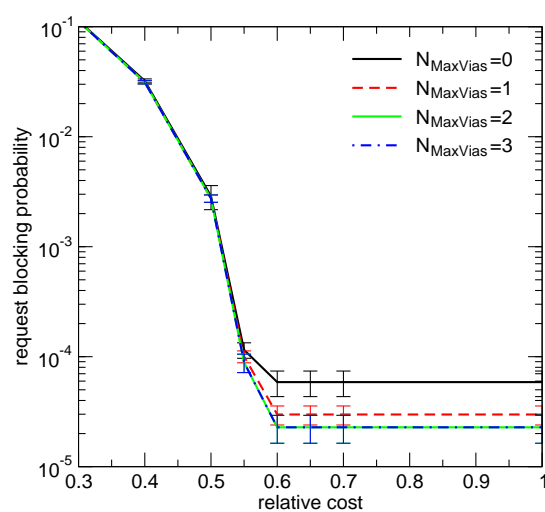

Figure 5. Influence of $N_{\mathrm{MaxVias}}$ 


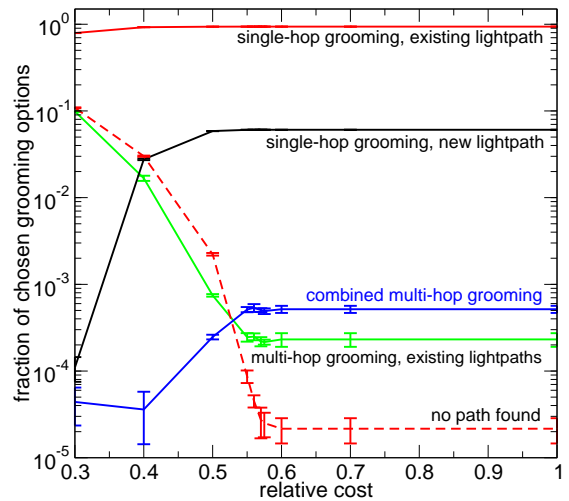

Figure 6. Path type distribution

lowers the request blocking probability by half an order of magnitude. Further increasing $N_{\text {MaxVias }}$ gives diminishing returns. For all $N_{\text {MaxVias }} \geq 2$ the results are identical due to the limited path length in the Germany network. Not shown are the results when varying $N_{\text {MaxAlt }}$, since there is only a negligible difference in performance.

Interesting enough, combined grooming greatly improves system performance (compare Fig. 5). However, in the interesting cost range of above 0.55 it is only used for approximately $0.1 \%$ of all connection requests, as can be seen in Fig. 6 . This picture shows the distribution of eventually selected grooming options. For low cost, an increased number of multi-hop paths on the SDH/SONET layer is chosen, whereas paths on the optical layer (i.e. setting up a new lightpath) become less frequently used. This is due to the lack of transponders at low cost, as the establishment of a new lightpath always requires a free transponder at the source and the destination node. For the same reason, combined grooming cannot be applied as often at low cost.

Several assumptions were made during the dimensioning of the transponders as described in section 4. Among others it was assumed that the through traffic in each node is negligible. To verify this hypothesis, the through traffic on the electrical layer $A_{\text {transit, } k}$ was measured at each node and normalized by the offered traffic $A_{\text {node, } k}$ at that node. The results can be seen in Fig. 7 , which shows the normalized through traffic for each node and five different cost points $(0.3,0.4, \ldots, 0.7)$. For low cost there is a significant amount of through traffic present in each node. However, this portion quickly drops below $2 \%$ for all, and even below $0.1 \%$ for most of the nodes as cost increases. In the interesting cost range of 0.5 and above, the through traffic accounts for less than $2 \%$ at each node, which makes the assumption of $A_{\text {transit, } k} \approx 0$ reasonable. It can also be observed that the through traffic expectedly is bigger in nodes which are located in the center of the network (labeled transit nodes in Fig. 7). 


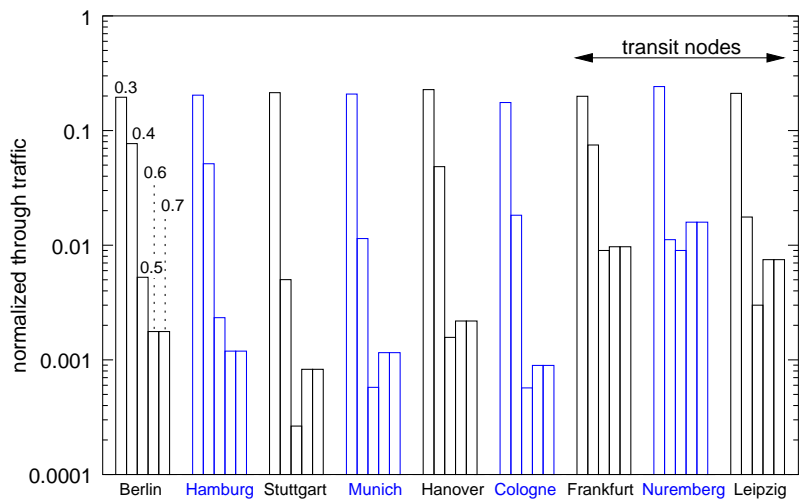

Figure 7. Normalized through traffic

In the course of dimensioning the transponders, another important assumption was that of only STM-16-connections being blocked. Fig. 8 shows the request blocking probability per connection request bandwidth for the two routing schemes WIR and PureOptical. Both routing schemes apparently favor connections with small granularities. This is obvious, since these connections can be routed more flexibly compared to connections that, for example, need an entire wavelength. For PureOptical, the blocking probability curves only differ by a scaling factor, whereas with WIR the blocking of STM-1- and 7-STM-1-connections is negligible for a relative cost of greater than 0.4 . The reason for a virtually blocking-free behavior of the network for STM-1- and 7-STM-1-connections with $W I R$ is that this routing scheme also allows for multi-hop grooming on the elec-
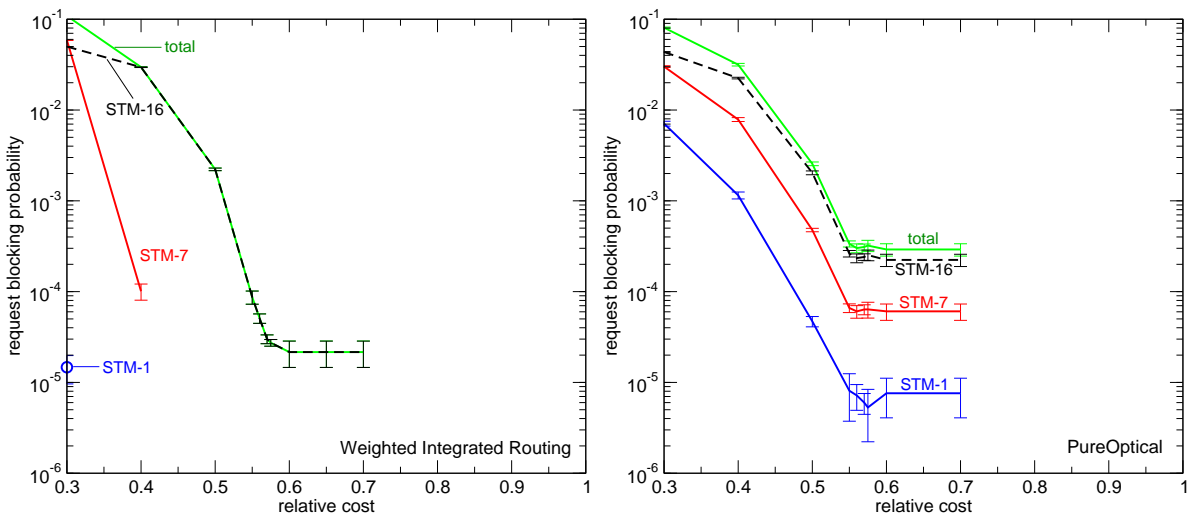

Figure 8. Request blocking probability per connection bandwidth 


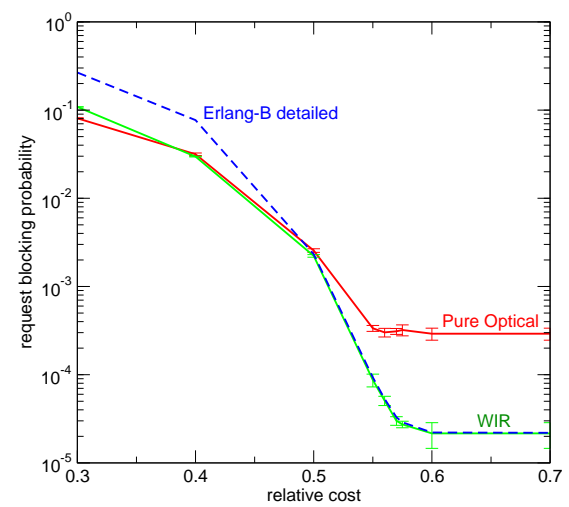

Figure 9. Lower limit of request blocking probability

trical layer and for combined multi-hop grooming, which makes it easy to find a path for connections with only small bandwidth demands.

It is desirable to predict the performance of a communication network before it is put into operation. In addition to the simulation studies, an analytical approach was taken to estimate the lower bound of the request blocking probability from the transponder blocking probability. Such an approach is likely to deliver a good estimate in the cost range of dominating transponder blocking. Using an iterative method with reduced load approximation, the request blocking probability caused by transponder blocking can be calculated for any node pair. These blocking probabilities are weighted with the corresponding traffic demand, delivering an estimate for the lower bound of the request blocking probability.

This estimate is shown in Fig. 9 (Erlang-B detailed). For the cost range of 0.5 and above, there is a good match between the estimate and the actual performance of the WIR-scheme. For lower cost, the estimate is not very accurate. The reason is that the presented Erlang-B-model does not take into account the different blocking behavior of different request granularities. In particular, the Erlang-B-model assumes a traffic mix of only STM-16-connections, resulting in a predicted request blocking probability which is too big at low cost.

It is astonishing that the estimate is even valid in the cost range where network blocking is expected to dominate. Further analysis leads to the insight that for a cost of 0.6 and above virtually all blocked connection requests originate from or end in small nodes at the edge of the network. The conclusion is that the optical layer of these nodes, i.e. the number of wavelengths $w$ connecting the nodes, is underdimensioned. Since the number of transponders $z$ might not exceed $w$, connection requests experience transponder blocking over the complete cost range if WIR is used. This interaction with network dimensioning is important and will be covered in future work. 


\section{Conclusion}

This work presented a new integrated multilayer routing scheme with reasonable complexity that outperforms non-integrated and even more complex integrated schemes. A major advantage for operators is the fact that no full topology information of the optical layer is needed. Instead, a small interface between the Routing Control Center and the electrical as well as the optical layer for probing rating information and signalling new connection requests or connection teardowns is sufficient. Simulations showed that improved performance can be achieved even without the need for a full integrated view of the network.

\section{Acknowledgement}

This work is the result of a diploma thesis done under supervision of Prof. Kühn at the Institute of Communication Networks and Computer Engineering at the University of Stuttgart. The author would like to thank the thesis tutors Christoph Gauger and Stefan Bodamer for their excellent support and valuable discussions in the course of this thesis.

\section{References}

1. ITU-T G.astn Draft V.0.3, "Architecture for the Automatic Switched Transport Network (ASTN)"

2. E. Modiano: "Traffic Grooming in WDM Networks", IEEE Communications Magazine, Vol. 39, No. 7, July 2001, pp. 124-129

3. K. Zhu, B. Mukherjee: "On-Line Approaches for Provisioning Connections of Different Bandwidth Granularities in WDM Mesh Networks", Proceedings of the Optical Fiber Communication Conference (OFC 2002), Anaheim

4. M. Kodialam, T.V. Lakshman: "Integrated Dynamic IP and Wavelength Routing in IP over WDM Networks", Proceedings of the IEEE INFOCOM 2001, New York City, Vol. 1, pp. 358-366

5. B. Rajagopalan, D. Pendarakis, D. Saha, R.S. Ramamoorthy, K. Bala: "IP over Optical Networks: Architectural Aspects", IEEE Communications Magazine, Vol 38, No. 9, September 2000, pp. 94-102

6. ITU-T Rec. G.707/clause 11, "Network Node Interface for the Synchronous Digital Hierarchy (SDH)", 2000

7. Institute of Communication Networks and Computer Engineering: "IND Simulation Library", www.ind.uni-stuttgart.de/INDSimLib

8. J. Späth: "Dynamic routing and resource allocation in WDM transport networks", Computer Networks, Vol. 32, No. 5, 2000, pp. 519-538

9. M.J. O'Mahony, D. Simeonidou, A. Yu, J. Zhou: "The design of a European optical network", IEEE Journal of Lightwave Technology, Vol. 13, No. 5, pp. 817-828, May 1995

10. K.-C. Lee, V.O.K. Li: "Routing and Switching in a Wavelength Convertible Optical Network", Proceedings of the IEEE INFOCOM 1993, Vol. 2, pp. 578- 585 